\title{
Permeability of packs of polydisperse hard spheres
}

\author{
Jérémie Vasseur $\odot,{ }^{1, *}$ Fabian B. Wadsworth $\odot,{ }^{2}$ Jason P. Coumans, ${ }^{2}$ and Donald B. Dingwell ${ }^{1}$ \\ ${ }^{1}$ Department of Earth and Environmental Science, Ludwig-Maximilians-Universität, Theresienstrasse 41, 80333 München, Germany \\ ${ }^{2}$ Department of Earth Sciences, Durham University, Durham, DH1 3LE, United Kingdom
}

(Received 9 February 2021; revised 27 May 2021; accepted 27 May 2021; published 25 June 2021)

\begin{abstract}
The permeability of packs of spheres is important in a wide range of physical scenarios. Here, we create numerically generated random periodic domains of spheres that are polydisperse in size and use lattice-Boltzmann simulations of fluid flow to determine the permeability of the pore phase interstitial to the spheres. We control the polydispersivity of the sphere size distribution and the porosity across the full range from high porosity to a close packing of spheres. We find that all results scale with a Stokes permeability adapted for polydisperse sphere sizes. We show that our determination of the permeability of random distributions of spheres is well approximated by models for cubic arrays of spheres at porosities greater than $\sim 0.38$, without any fitting parameters. Below this value, the Kozeny-Carman relationship provides a good approximation for dense, closely packed sphere packs across all polydispersivity.
\end{abstract}

DOI: 10.1103/PhysRevE.103.062613

\section{INTRODUCTION AND BACKGROUND}

Understanding the relationship between the microstructure of porous solids and their bulk properties is central to general descriptions of randomly assembled heterogeneous materials [1]. In particular, the hydraulic properties of packs of solid particles are fundamental to a wide range of applied problems from hydrology and carbon capture and storage [2], hydrocarbon transport and accumulation in sedimentary sequences [3,4], soil science [5], liquefaction [6], volcanic eruptions [7,8], and ceramic synthesis [9], among others. At low Reynolds number, Darcy's law describes the proportionality between a fluid pressure gradient $\nabla p$, and the resultant average fluid velocity $\langle u\rangle[10]$ - where a proportionality factor is the ratio between the fluid viscosity $\mu_{f}$ and the permeability of the porous solid $k$ :

$$
\nabla p=-\frac{\mu_{f}}{k}\langle u\rangle .
$$

Therefore, a fundamental aim of research into porous media is to build predictive constitutive models for how $k$ varies with the porosity $\phi$. The form of that relationship, $k(\phi)$, is often found to be specific to individual microstructure types $[1,8]$.

Perhaps the most widely used permeability model for real packed particulate or granular media is the so-called Kozeny-Carman equation $[11,12]$ together with extensions or adaptations thereof $[13,14]$. These approaches are successful

\footnotetext{
*j.vasseur@lmu.de
}

Published by the American Physical Society under the terms of the Creative Commons Attribution 4.0 International license. Further distribution of this work must maintain attribution to the author(s) and the published article's title, journal citation, and DOI. in part because they typically involve the use of a dimensionless scaling factor $C$, which can be determined empirically, and which allows a degree of freedom in fitting the equation to data across a range of microstructure types. The KozenyCarman models are given by a general form [15],

$$
k=\frac{\phi^{3}}{C s^{2}},
$$

where $s$ is the specific surface area. For systems of hard monodisperse spheres of radius $R, s=3(1-\phi) / R$. Thus Eq. (2) may be expanded into the more commonly found form $k=\phi^{3} R^{2} /\left[9 C(1-\phi)^{2}\right]$ [16]. While Eq. (2) is powerful and used widely, the observation that $C$ is not universal to all microstructures renders its value specific to each microstructure type. Torquato [1] suggests that $C=5$ facilitates a good fit to $k(\phi)$ across many porous media. We note, however, that in some cases $C$ is found to be variable and is composed as a function of porosity itself [15].

In contrast, for the case of packs of spheres arranged in cubic arrays, there are theoretical approaches that require no empirical adjustment within their field of validity $[17,18]$ and for which validation against data exists $[8,19]$. These models are in the form of asymptotic series expansions of Stokes scaling for permeability $k_{s}$ in the dilute limit $\phi \rightarrow 1$, with the aim of extending $k_{s}$ to intermediate $\phi$. Expansions of this type have the form $[1,18]$

$$
k=k_{s}\left[\sum_{i=0}^{30} c_{i}\left(\frac{1-\phi}{\phi_{m}}\right)^{i / 3}\right]^{-1},
$$

where $c_{i}$ are the expansion coefficients [18] for indices $i$ (given by Sangani and Acrivos [18] up to $i=30$ ), and $\phi_{m}$ is the porosity at maximum packing such that $\phi<\phi_{m}$ is physically inaccessible (for cubic pack types $\phi_{m}$ is well defined). For simple, body- and face-centered cubic lattice arrangements, the porosity at maximum packing is given by 
(a)

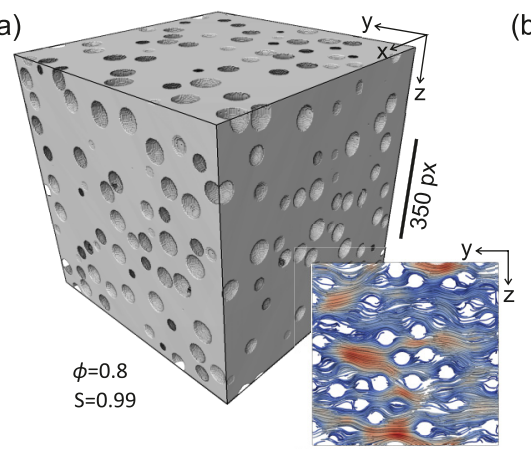

(d)

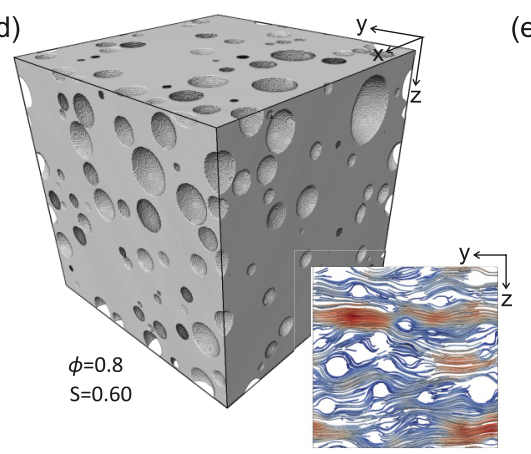

(b)

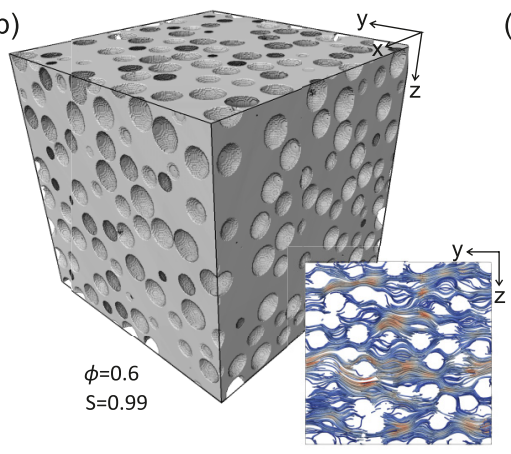

(c)

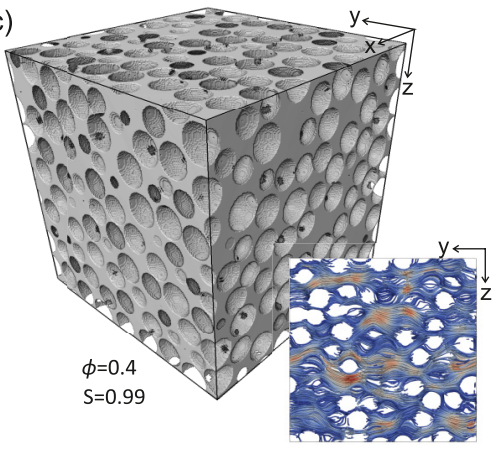

(e)

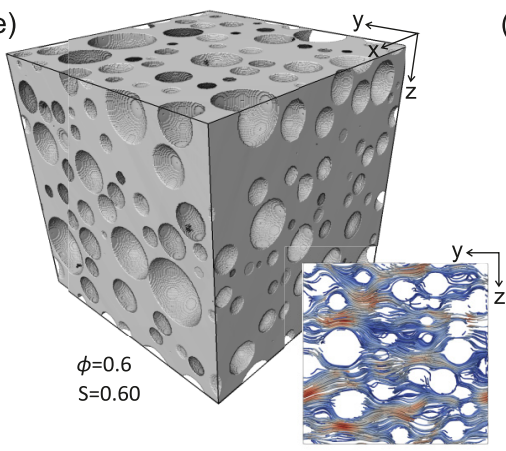

(f)

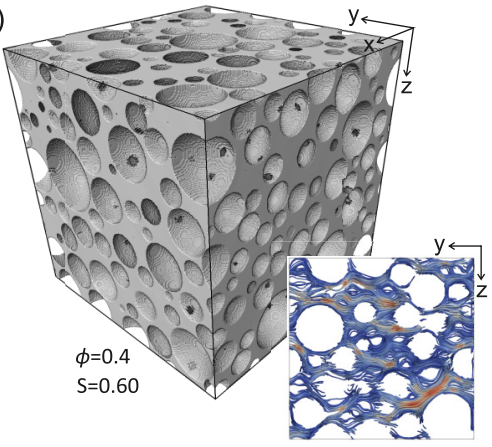

(g)

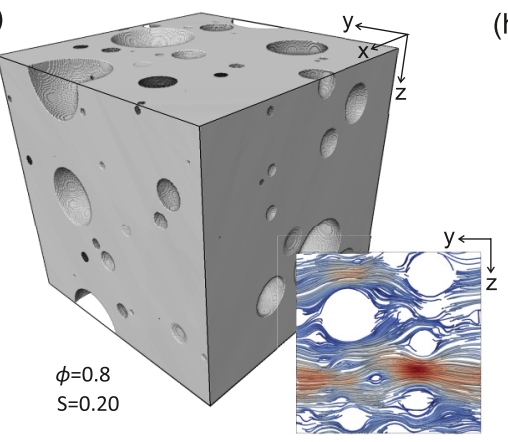

(h)

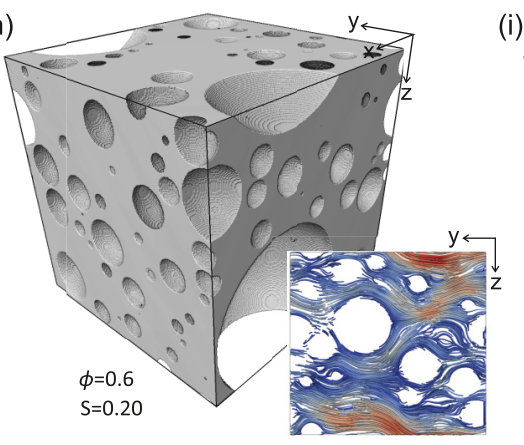

(i)

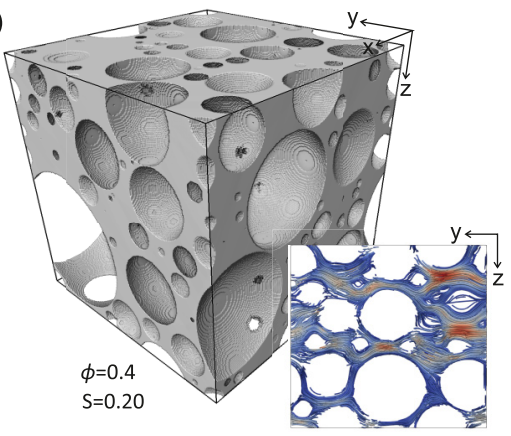

FIG. 1. Rendered examples of numerical domains used herein at polydispersivity $S=0.99$ (a)-(c), $S=0.6$ (d)-(f), and $S=0.2$ (g)-(i). In all cases, $N=1000$, the gray shaded region represents the pore phase (with volume fraction $\phi$ ) and the sphere phase is transparent. Examples are shown at $\phi=0.8$ (a), (d), (g), $\phi=0.6$ (b), (e), (h), and $\phi=0.4$ (c), (f), (i). Inset: example 2D slices through the full 3D distribution of fluid speeds output from the lattice-Boltzmann simulations with a pressure gradient applied in the $y$ direction. Warm colors denote high speeds compared with cold colors (note that the colors are given for relative comparison and that the absolute values of the speed are not relevant).

$\phi_{m}=1-\pi / 6, \phi_{m}=1-\sqrt{3} \pi / 8$, and $\phi_{m}=1-\sqrt{2} \pi / 6$, respectively. Equation (3) has been shown to only give accurate results for $(1-\phi) / \phi_{m}<0.85$ and deviations are to be expected for closely packed arrays. It should be noted that, while Sangani and Acrivos [18] derive an expression for the drag coefficient $K$, it can be shown that $k=k_{s} / K[1,19]$. The Stokes permeability $k_{\mathrm{s}}$ is

$$
k_{s}=\frac{2(1-\phi)}{s^{2}} .
$$

For a dilute bed of identical spheres, Eq. (4) can be expanded to recover the well-known Stokes result $k_{s}=$ $2 R^{2} /[9(1-\phi)][1]$. As noted above, the asymptotic series expansions [Eq. (3)] are validated for spheres packed in cubic lattice arrangements, but it is as yet not clear how applicable this approach is for packs of randomly assembled spheres or polydisperse sphere packs.

Here, we use a sphere pack generation algorithm to create populations of polydisperse hard spheres and a numerical fluid flow simulation tool to determine the Darcian permeability of the packs. We seek to find a simple solution for the permeability of packs of hard spheres that explicitly accounts for polydispersivity of the sphere sizes across as wide a range of $\phi$ as possible.

\section{METHODS AND SAMPLE GEOMETRIES}

Here we describe the numerical methods we used to (1) create the domains of polydisperse sphere packs at a wide range of porosity, and (2) simulate the fluid flow that is used to compute the permeability of the packs.

\section{A. Numerically generated samples}

The widely used random sequential adsorption (RSA) method for producing numerical packs of spheres [20] is effective at producing low-density packs at high $\phi$, but is usually 

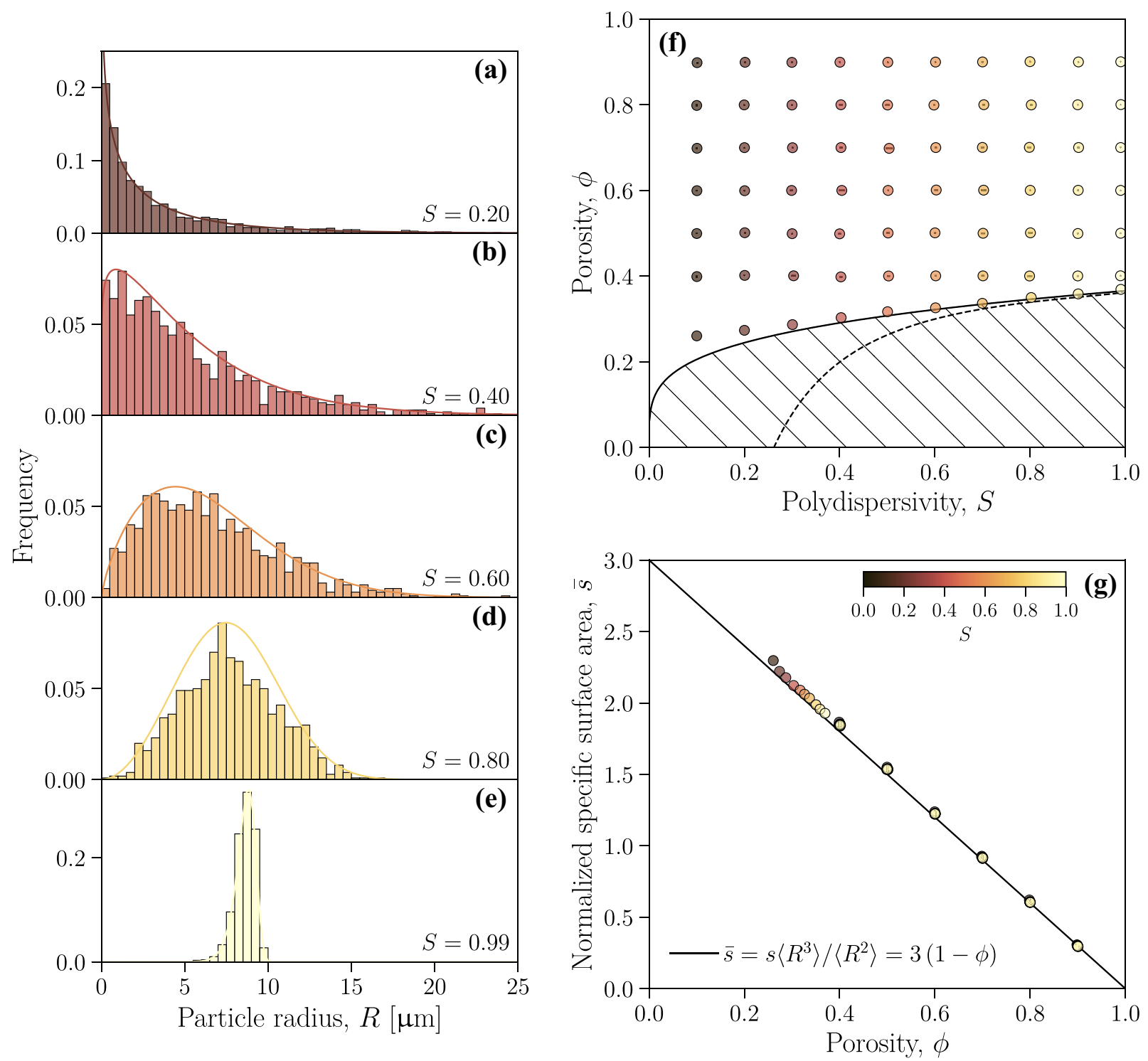

FIG. 2. Basic properties of the sphere packs used here for $L=300 \mu \mathrm{m}$. (a)-(e) The size distribution of the sphere packs used here for $N=1000$ and $\phi=0.9$ and for (a) $S=0.2$, (b) $S=0.4$, (c) $S=0.6$, (d) $S=0.8$, and (e) $S=0.99$. The measured distributions are given by the histograms binned at $0.5 \mu \mathrm{m}$ bin sizes in $R$, and the continuous curves represent the target Weibull distribution of the algorithm used with shape parameter (a) $\alpha=0.75$, (b) $\alpha=1.14$, (c) $\alpha=1.71$, (d) $\alpha=2.94$, and (e) $\alpha=17.04$. (f) The porosity as a function of polydispersivity of the packs produced here. The hatched region represents an empirical approximation for the inaccessible region at low $\phi$ below $\phi_{m}$ given here by $\phi_{m} \approx \phi_{\mathrm{rcp}}=\phi_{\mathrm{rcp}}^{*} S^{1 / 4}$ with $\phi_{\mathrm{rcp}}^{*}=0.366$. This curve is given to guide the eye and is not anticipated to be rigorous. The dashed curve is the empirical formula given by Desmond and Weeks [27], which breaks down for $S \leqslant 0.26$. (g) The specific surface area as a function of porosity used to validate the pack resolution. Each data point in (f), (g) represents the arithmetic mean across $N$ and the error bars give $1 \sigma$ about that mean.

unable to produce sphere packs at higher density [21,22]. Higher densities can, however, be readily achieved if the RSA method is used to produce relatively low-density packs, which are then numerically stepwise compressed [23]. While this method can extend sphere pack densities, it cannot generate jammed packs. Densities that approach jammed states can instead be achieved with a molecular dynamics approach [24] applied to spheres [25]. This approach begins with a fixed number of particles $N$ at zero sphere volume in the domain of a given edge length $L$. The spheres are assigned random velocity vectors and the domain has periodic boundaries. At each iteration step, a growth law is applied such that the volume of the spheres increases with time at a fixed rate, and particle-particle collisions are processed to recalculate velocities where necessary.

In this work we use the molecular dynamics approach. Specifically, we use the event-based numerical algorithm by Ghossein and Lévesque [21]. Our implementation differs most substantially from theirs in that the particle radius growth rates $r$ are drawn from a Weibull distribution such that the probability that a particle has a radius growth rate between $r$ and $r+d r$ is given by $p(r)=\alpha r^{\alpha-1} \exp \left(-r^{\alpha}\right)$, where $\alpha$ is a 

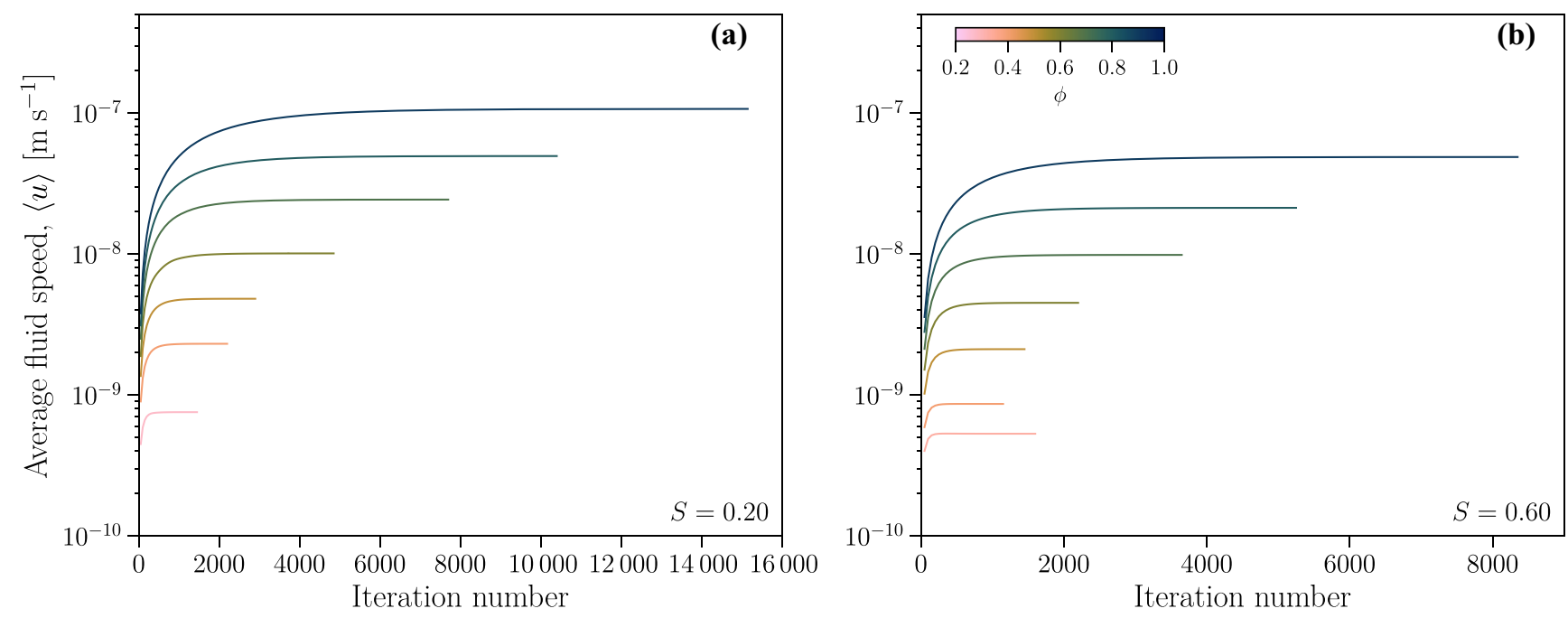

FIG. 3. The average fluid speed as a function of iteration number from the numerical simulation of fluid flow through the packs generated here. We show the results for all $\phi$ for domains at $N=1000$ and $L=300 \mu \mathrm{m}$, for two $S$ : (a) $S=0.2$ and (b) $S=0.6$. The fluid speed at steady state (large iteration number) is used to compute $k$. Here we show curves for flow in one principal direction only.

constant shape parameter. In this manner we obtain particles with radii following the same distribution, i.e., $p(R)$.

In order to create packs in which the sphere size distribution is polydisperse, we define a polydispersivity parameter $S$ $[1]$,

$$
S=\frac{\langle R\rangle\left\langle R^{2}\right\rangle}{\left\langle R^{3}\right\rangle},
$$

for which $\left\langle R^{j}\right\rangle$ is the $j$ th moment of the distribution of sphere sizes with $\langle R\rangle,\left\langle R^{2}\right\rangle$, and $\left\langle R^{3}\right\rangle$ corresponding to the mean, variance, and skewness, respectively. $S$ can be thought of as the ratio of the specific surface area of a polydisperse system to that of a monodisperse system with the same $\phi$ and for which the mean of the polydisperse system is the monodisperse radius. Therefore, $S=1$ is the monodisperse limit regardless of the values of $\left\langle R^{j}\right\rangle$ and $S=0$ is an infinitely polydisperse limit. Torquato [1] provides examples of $S(\phi)$ for the Schulz and log-normal distributions in which the distribution control parameter is varied continuously; similar behavior arises with the Weibull distribution used herein where $\alpha$ is our distribution control parameter. We vary $S$ across a wide range and then compute $\alpha$ accordingly to result in each distribution of sizes. For the probability density function $p(R)$ defined here, $\left\langle R^{j}\right\rangle=\Gamma(1+j / \alpha)$ where $\Gamma$ is the gamma function.

In Fig. 1 we show rendered examples of the pore phase between the spheres (the spheres are made invisible because the phase of interest is the pore phase with volume fraction $\phi)$ in each domain. In Fig. 2 we show the output sphere size distributions $f(R)$ measured in the domains produced together with the corresponding Weibull target distribution [Figs. 2(a)-2(e)] for each value of $\phi$. We generate samples in two steps-first, we generate samples at high $\phi$ covering $0.1 \leqslant S \leqslant 0.99$ and $0.4 \leqslant \phi \leqslant 0.9$, as well as $100 \leqslant N \leqslant$ 2000. In the second step, we generate samples close to their close packing state [Fig. 2(f)]. Whereas for cubic packs of spheres, there is a formal maximum packing $\phi_{m}$ [see Eq. (3)], for random and heterogeneous packs of spheres, this is not well defined [26]. However, we have a porosity limit below which our algorithm cannot generate a pack, which we refer to as the random close-pack porosity $\phi_{\text {rcp }}$.

In Fig. 2(f) we give an empirical form for the random close pack window $\phi_{\text {rcp }}(S)$ where $\phi<\phi_{\text {rcp }}$ is inaccessible. We use $\phi_{\mathrm{rcp}}=\phi_{\mathrm{rcp}}^{*} S^{1 / 4}$ where $\phi_{\mathrm{rcp}}^{*}$ is the porosity corresponding to the random close pack value for monodisperse spheres (i.e., $\left.\phi_{\text {rcp }}^{*}=0.366\right)$. We note that this parametrization is not rigorous [26] and is simply to guide the reader in Fig. 2(f). Other authors have proposed an empirical formula to describe this random close pack window [27]. However, their description is valid only for $S>0.26$ [Fig. 2(f)].

As a verification step to test the accuracy and resolution of our numerically generated samples, we use a marching cubes algorithm to find the surface area of the spheres [28,29]. Normalizing the surface area to the domain volume gives the specific surface area, $s$. The specific surface area is analytically found from [1]

$$
\begin{aligned}
& s=\frac{3(1-\phi)}{R}, \\
& s=3(1-\phi) \frac{\left\langle R^{2}\right\rangle}{\left\langle R^{3}\right\rangle}=3(1-\phi) \frac{S}{\langle R\rangle},
\end{aligned}
$$

where Eqs. (6a) and (6b) are for monodisperse and polydisperse distributions of hard spheres, respectively. Equation (6) can be compared with our results directly. We confirm that we reproduce the expected specific surface areas to within 99.7\% accuracy [Fig. 2(g)]; using the normalized functions $\bar{s}=s R=3(1-\phi)$ and $\bar{s}=s\left\langle R^{3}\right\rangle /\left\langle R^{2}\right\rangle=3(1-\phi)$, we arrive at a universal description of the specific surface internal to packs of hard spheres for monodisperse and polydisperse packs, respectively. This gives us confidence that our numerical resolution of the sphere surfaces is sufficient across all $S$ and $\phi$ and leads us to use $L=300 \mu \mathrm{m}$ (see Sec. II B). 

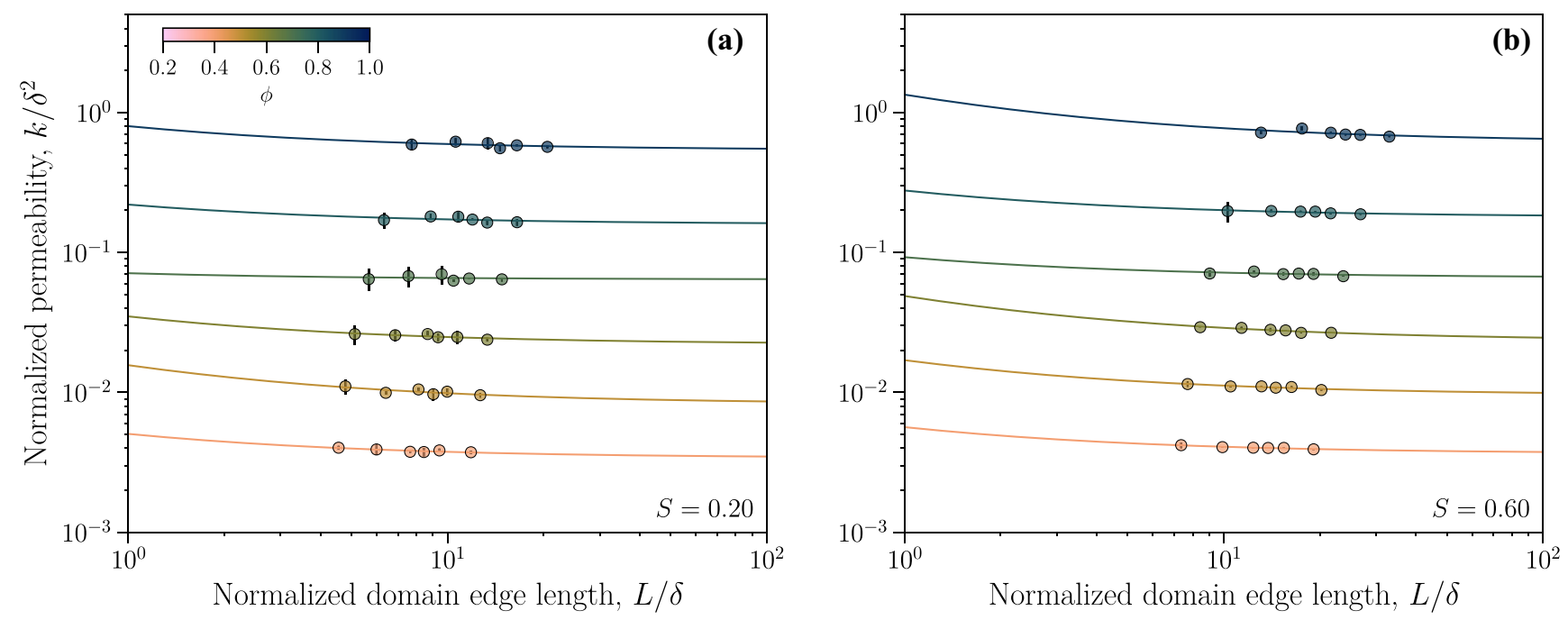

FIG. 4. The effect of particle number $N$ on the normalized permeability $k / \delta^{2}$. Each data point represents the arithmetic mean of the permeability in the three principal directions and the error bars give $1 \sigma$ about that mean. We show the fits (solid lines) to Eq. (7), based on a semiempirical function proposed by Matyka et al. [32]. We use this to extrapolate to large $L / \delta$ beyond the region where domain size plays a role. In subsequent analysis, only these single extrapolated values are used (see text and Fig. 6). We show the results for two $S$ : (a) $S=0.2$ and (b) $S=0.6$.

\section{B. Numerical simulations}

The numerically generated samples (Sec. II A) are binary (i.e., two phase). We define the spheres as the "solid phase" and the space between the spheres as a fluid phase. LBflow is a lattice-Boltzmann fluid flow simulator [19,30], which has been calibrated for body-centered cubic [19] and simple-cubic [31] packs of spheres. The LBflow code discretizes the pore phase of interest into a lattice of fluid nodes (one fluid node per voxel). Packets of fluid mass are propagated through the lattice positions with numerical time $t$ and undergo collisions using a $\mathrm{D}_{3} \mathrm{Q}_{15}$ lattice arrangement. We apply a numerical pressure gradient of $\nabla p=0.01 \mathrm{~Pa} \mathrm{~m}^{-1}$ and an input fluid viscosity of $\mu_{f}=1.8205 \times 10^{-5} \mathrm{~Pa} \mathrm{~s}$ and density of $\rho_{f}=$ $1.2047 \mathrm{~kg} \mathrm{~m}^{-3}$ corresponding to that of air at ambient pressure and temperature conditions. We employ a steady-state criterion such that the average fluid speed across the lattice must not vary by a factor of $10^{-5}$ twice consecutively over 50 iteration steps [30].

The ultimate output of the LBflow model is the spatial distribution of fluid velocity vectors at steady state. Representative two-dimensional (2D) slices through the full three-dimensional (3D) velocity vector distribution are shown as insets to Fig. 1. The average fluid velocity in the direction of the applied pressure gradient is given by $\langle u\rangle=u \phi$ and is used as an input to Darcy's law [Eq. (1)] to compute the permeability for a given domain. In Fig. 3 we demonstrate how the average fluid speed relaxes to a steady-state value as the simulation approaches the convergence criterion for $S=0.2$ [Fig. 3(a)] and $S=0.6$ [Fig. 3(b)]. It is the steadystate value of $\langle u\rangle$ that we use in Eq. (1) to find $k$.

The input conditions for $\mu_{f}$ and $\rho_{f}$ are selected to ensure creeping flow at low Reynolds number Re and low Mach number Ma. Using an average approach, we define $\operatorname{Re}=\langle u\rangle \rho_{f} L / \mu_{f}$ and $\mathrm{Ma}=\langle u\rangle / v_{s}[7,8,19,30]$, where $v_{s}$ is the speed of sound in the fluid medium (see [19,30]). For our simulations we find that $10^{-8}<\operatorname{Re}<10^{-5}$ and $10^{-12}<$ $\mathrm{Ma}<10^{-9}$. The invariance of our result with variations in Re and Ma supports a conclusion that our results do not depend on the fluid properties we choose here and that all simulation results are in the same regime for which Eq. (1) is valid.

When using lattice-Boltzmann fluid flow simulations, important considerations include (1) that the flow in the small gaps is well resolved, and (2) that the domain is representative of continuum parameters (including the permeability). The former is of particular concern when investigating fluid flow for packs of particles close to their random close packing. To test these two issues, we follow the approach of Matyka et al. [32] (cf. their Sec. IV D) for which we use $\delta=\left\langle R^{3}\right\rangle /\left\langle R^{2}\right\rangle=\langle R\rangle / S$ as our normalization length scale, consistent with Torquato [1].

We vary the number of particles in the domain $N$, which affects the sphere sizes and therefore the discretization resolution of our domains such that $\delta$ is varied over a wide range. For a given $L$, that results in a range of $L / \delta$ over which the analysis is performed. We find that at a combination of low $\phi$ and high $S$, our results do not depend strongly on $L$, whereas at high $\phi$ and/or low $S$, there is an apparent dependence (Fig. 4). To account for this, we normalize the permeability $k / \delta^{2}$ and fit

$$
\frac{k}{\delta^{2}}=\kappa_{\infty}-\kappa\left(\frac{L}{\delta}\right)^{-2 / 3}
$$

where $\kappa_{\infty}$ and $\kappa$ are dimensionless constants. Matyka et al. [32] use the same approach to extrapolate their permeability results to large $L / \delta$ at which they can be sure that the volume is representative (i.e., the value of $\kappa_{\infty}$ ). Here we apply this approach and in Fig. 4 we show the fits to this approach [Eq. (7)] for $S=0.2$ [Fig. 4(a)] and $S=0.6$ [Fig. 4(b)]. 


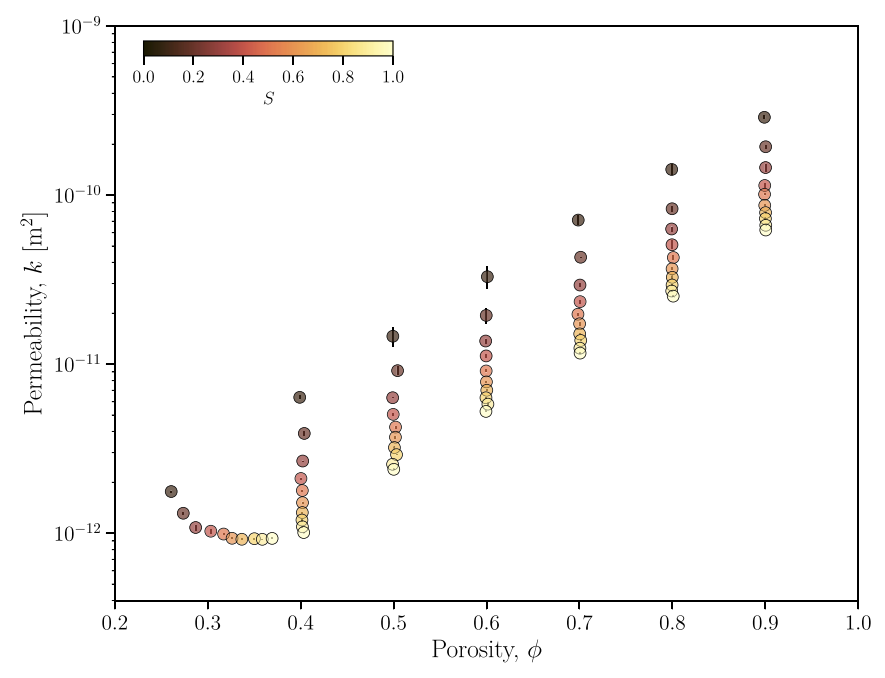

FIG. 5. The raw permeability $k$ as a function of porosity $\phi$ for all polydispersivity $S$ for $N=1000$ and $L=300 \mu \mathrm{m}$. Each data point represents the arithmetic mean of the permeability in the three principal directions and the error bars give $1 \sigma$ about that mean.

\section{RESULTS AND MODEL ANALYSIS}

We find that the permeability depends on the porosity $\phi$, the polydispersivity $S$, and the sphere sizes via $s$. At fixed $\phi$, decreasing $S$ (moving to more polydisperse distributions of spheres) has the effect of increasing $k$. Similarly, at fixed $S$, decreasing $\phi$ has the effect of decreasing $k$ (Fig. 5). Most of our results for $k(\phi, S)$ are for a given fixed $\langle R\rangle$. However, in order to generalize to arbitrary sphere sizes, we recast the existing models in dimensionless form. This is achieved by normalizing each model (see Sec. I) by a form of $k_{s}$ that is adapted to account for polydisperse sphere sizes by introducing $s$ from Eq. (6b). Here we give three equivalent variants of this polydisperse $k_{s}$ :

$$
k_{s} \approx \frac{2\left\langle R^{3}\right\rangle^{2}}{9(1-\phi)\left\langle R^{2}\right\rangle^{2}}=\frac{2\langle R\rangle^{2}}{9(1-\phi) S^{2}}=\frac{2 \delta^{2}}{9(1-\phi)} .
$$

In Eq. (8), we state that $k_{s}$ is only approximately equal to the polydisperse extensions, because we note that Torquato and $\mathrm{Lu}[33]$ found that strictly speaking, $k_{s}=$ $2\left\langle R^{3}\right\rangle /[9(1-\phi)\langle R\rangle]$. Nevertheless, we follow Torquato and $\mathrm{Lu}$ [33] in using Eq. (8) as the most effective scaling for polydisperse systems.

\section{A. Dimensionless models}

Taking a dimensionless permeability as $\bar{k}=k / k_{s}$, we can render each model dimensionless. For the asymptotic cubic expansion models [18,19] Eq. (3) gives

$$
\bar{k}=\left[\sum_{i=0}^{30} c_{i}\left(\frac{1-\phi}{\phi_{m}}\right)^{i / 3}\right]^{-1},
$$

and for the Kozeny-Carman model $[11,12,15]$ Eq. (2) gives

$$
\bar{k}=\frac{\phi^{3}}{2 C(1-\phi)} \text {. }
$$

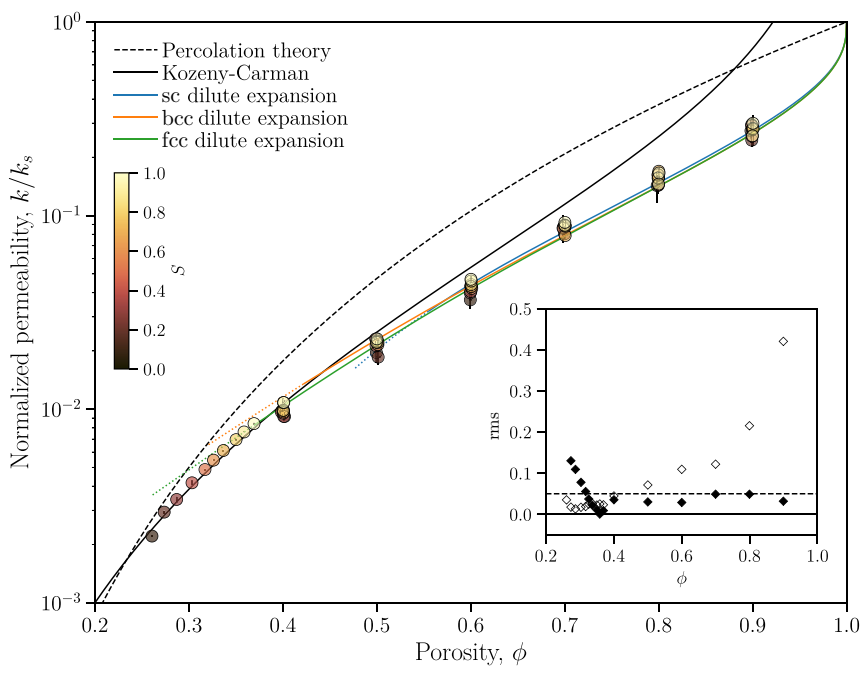

FIG. 6. The scaled results given as $\bar{k}=k / k_{s}$ as a function of $\phi$. Simple-cubic (sc), body-centered cubic (bcc), and face-centered cubic (fcc) refer to the three variants of the asymptotic expansions of the Stokes scaling for permeability for cubic lattice arrangements of particles [Eq. (9)]. The Kozeny-Carman model [Eq. (10)] is shown for $C=5$ and the percolation model [Eq. (12)] for $e=4$.4. Each data point represents the arithmetic mean of the permeability in the three principal directions and the error bars give $1 \sigma$ about that mean. Inset: the rms statistic applied to compare the relative distance between the data for each $\phi$ and either the fcc model (filled points) or the Kozeny-Carman model (unfilled points); see text for details.

\section{B. Comparison with data}

We apply the same transformation $\vec{k}=k / k_{s}$ [using Eq. (8)] to our data. This gives a universal scaled result shown in Fig. 6. To account for domain size effects (see Sec. II B), in this scaling we use the fitted $\kappa_{\infty}$ from Eq. (7), such that $\bar{k}=k / k_{s}=9 \kappa_{\infty}(1-\phi) / 2$ and the result is scale independent. Across all values of $S$ the data collapse to a single $\bar{k}(\phi)$ trend. This behavior persists to close to a maximum packing at low $\phi$. We conclude that $k_{s}$ adapted to account for polydisperse sphere sizes [Eq. (8)] is an appropriate universal scaling for hard sphere packs.

The results shown in Fig. 6 allow the general forms of each dimensionless model presented in Sec. III A to be compared with the collapsed universal dataset. We find that the expansions of $k_{s}$ for cubic packs of monodisperse spheres [Eq. (9)] provide a generally good description of $\bar{k}(\phi)$ even though the random and polydisperse nature of our simulation domains invalidates the exact microstructure on which the expansions were based (i.e., cubic lattice arrangements [18]). The goodness of fit of Eq. (9) is apparently independent of cubic lattice type (e.g., simple, face-centered, or body-centered) at high $\phi$. At intermediate $\phi$, minor differences between the solution for each lattice type emerge. First, the simple and body-centered packing types have $\phi_{m}$ values above the minimum $\phi$ studied here (i.e., $\phi_{\text {rcp }}$ ), and so cannot formally provide a good description across all $\phi$. Additionally, even in the region $0.32 \lesssim$ $\phi \lesssim 0.45$ where both the body-centered and face-centered cubic solutions are valid, the face-centered cubic packing solution provides a marginally better description of our $\bar{k}(\phi)$ data. We conclude that the form of Eq. (9) with coefficients 
$c_{i}$ for face-centered cubic packs of spheres is a tenable model for $k(\phi, S)$ when $S$ is incorporated via $k_{s}$ adapted in Eq. (8).

The goodness of fit of Eq. (9) to our data (without introducing any adjustable parameter) is surely appealing. For random sphere packs with low $S$ near their random close maximum packing, our data deviate from the descriptions given by Eq. (9), and $\bar{k}$ is lower than predicted by up to a factor of 1.09. In any real pack of particles for which $k(\phi)$ could be measured, the system will necessarily be jammed at or close to $\phi_{\text {rcp }}$, and so it is particularly in this region that model fidelity is required. The other model tested here is given by Eq. (10) and represents the more widely used model approach to predicting $k(\phi)$ for sphere packs. While the Kozeny-Carman model [Eq. (10) with $C=5$ ] does not match the data well across all $\phi$ (failing particularly in the dilute limit $\phi \rightarrow 1$ ) it provides a reasonable match to the maximally packed data at low to intermediate $\phi$. We find that this model matches the maximally packed data for $C=5$ to within $98.3 \%$ accuracy in the region $0.27<\phi<0.38$. If we let $C$ act as a fitting parameter and use a least-squares regression minimization, we find that the best fit in this region of $\phi$ is $C=4.83 \pm 0.06$, close to the proposed $C=5$ [1]. We note that there is no value of $C$ for which the Kozeny-Carman functional form can outperform Eq. (9) across all $\phi$.

\section{DISCUSSION}

Our results show that the expansions of $k_{s}$ from $\phi \rightarrow 1$ to intermediate $\phi$ [Eq. (9)] provide a good description of the data at $\phi>0.38$ when $k_{s}$ is adjusted to account for polydisperse sphere packs [Eq. (8)]. This description is appealing because it involves no empirical adjustment. The fact that a model based on cubic arrangements of spheres appears to work even for random distributions of spheres leads us to conclude that the arrangement of the particles in the pack is less important than their geometry (e.g., spheres in this case).

We can think of the comparison between data and models (Sec. III B) as yielding two apparent microstructural regimes. First, for packs of polydisperse spheres at $\phi>0.38$ or packs of monodisperse spheres at any $\phi$, the cubic lattice packing law [Eq. (9)] outperforms other models. For packs of polydisperse spheres at $\phi<0.38$, the Kozeny-Carman law [Eq. (10)] provides the best description of the data with $C=5$. Indeed, we note that there is crossover in the range $0.35<\phi<0.55$ in which both models appear to provide a reasonable description of the data. We reiterate this crossover by plotting the root mean square statistic rms $=\sqrt{\sum\left(\bar{k}-\bar{k}^{\prime}\right)^{2} / n}$ for each $\phi$, where $\bar{k}$ is the measured value, $\bar{k}^{\prime}$ the predicted value from the model being considered [Eqs. (9) and (10)], and $n$ the number of data points. We show $\operatorname{rms}(\phi)$ in the inset to Fig. 6 , which demonstrates that the cubic packing models (using the face-centered cubic pack as a benchmark model with the lowest $\phi_{m}$ ) have a rms value lower than the Kozeny-Carman model in the region $\phi>0.4$. Conversely, this demonstrates that the Kozeny-Carman model has a lower rms value in the region $\phi_{\text {rcp }}<\phi<0.4$. The Kozeny-Carman model and variants thereof are typically applied at the close packed values of $\phi$ where spheres or particles are jammed and therefore support direct measurement $[4,8,31,34-36]$. The fact that this model captures the data we provide here for low $S$ and low $\phi$ leads us to propose that the microstructure of randomly distributed maximally packed polydisperse spheres deviates significantly from cubic arrangements of hard spheres.

Percolation laws have the form $k \propto \phi^{e}$, where $e$ is a percolation exponent $[4,34,37]$. These laws often involve a percolation transition porosity or "percolation threshold" $\phi_{c}$, below which $k=0$. A general form of these laws is

$$
k=k_{r}\left(\phi-\phi_{c}\right)^{e},
$$

where $k_{r}$ is a reference permeability. $k_{r}$ is a function of $s$, which, in many cases, is also taken to be a function of $\phi[4,34]$. Rigorous theoretical constraints have been placed on $e$ for different microstructural geometries [37,38], and simulations and experiments have constrained $\phi_{c}[8,39,40]$. It follows that there exist forms of these percolation models that appear to be effective without the requirement of fitting to empirical constants. Nevertheless, these laws break down at high $\phi[8,31]$, and are only clearly valid for overlapping particles. These percolation laws are most applicable to systems of overlapping spheres $[8,34]$ or systems where a nonoverlapping hard sphere description is not valid (e.g., data for natural rock samples) [41].

Acknowledging that there is no percolation threshold for hard spheres, we set $\phi_{c}=0$ for the percolation model and take $k_{r}=k_{s}$ (which with $\phi_{c}=0$ is the same as $k_{r}$ given in Martys et al. [34]) such that Eq. (11) yields

$$
\bar{k}=\phi^{e} \text {. }
$$

We note that this percolation model given in Eq. (12) does not provide a reasonable match to the maximally packed data in Fig. 6 at any $\phi$ except for the lowest values, which is for the most polydisperse systems tested. While Eq. (12) has been used successfully to describe the permeability of packs of overlapping spheres, we conclude that it provides a poor description of packs of polydisperse hard spheres when compared with the other models tested here.

Analysis of $C$ in terms of hydraulic drag (or friction) and tortuosity in controlled experiments of particles of a given shape at varying $\phi$ is a reasonable way to approach more complex porous media. The extension of the use of the percolation model [Eq. (12)] to these kinds of packs of porous media would likely require similar empirical adjustments.

\section{CONCLUDING REMARKS}

We created numerical domains of polydisperse spheres at a range of porosities. We used numerical simulations to constrain the permeability of these packs. These data are then used to assess the efficacy of existing models for permeability as a function of porosity in the case of high polydispersivity of particle sizes. We find that different model approaches have fields of efficacy, with cubic pack models providing a broadly good description of the permeability across most porosity and polydispersivity. We specifically investigate the high polydispersivity case at low porosity-a case typical of natural particle packs - and find that the Kozeny-Carman description is more effective in this regime. We propose that there is a switchover between monodisperse or loose packs for which the cubic packing laws provide a good description, to the dense packs of polydisperse particles, for which the 
Kozeny-Carman model provides a superior fit. We propose that this framework represents an extension of the existing understanding of the permeability of packs of spheres to polydisperse distributions of spheres arranged randomly.

\section{ACKNOWLEDGMENTS}

This work was funded by the European Research Council (Advanced Grant No. EAVESDROP 834225) to D.B.D. We acknowledge the Natural and Environment Research Council (U. K.) under Grant No. NE/N002954/1 (PI: Edward W. Llewellin), which facilitated the contribution of J.C. to this work and the Centre for Advanced Studies (at the LudwigMaximilians-Universität) who provided a visiting fellowship to F.W. We thank the Leibniz Supercomputing Centre of the Bavarian Academy of Sciences and Humanities (Garching bei München, Germany) for computational support. We are grateful to Eloïse Bretagne for fruitful discussion.
[1] S. Torquato, Random Heterogeneous Materials: Microstructure and Macroscopic Properties, Vol. 16 (Springer Science \& Business Media, New York, 2002).

[2] S. A. Mathias, J. G. Gluyas, G. J. González Martínez de Miguel, S. L. Bryant, and D. Wilson, On relative permeability data uncertainty and $\mathrm{CO}_{2}$ injectivity estimation for brine aquifers, Int. J. Greenhouse Gas Control 12, 200 (2013).

[3] T. Bourbie and B. Zinszner, Hydraulic and acoustic properties as a function of porosity in Fontainebleau sandstone, J. Geophys. Res. 90, 11524 (1985).

[4] F. B. Wadsworth, J. Vasseur, B. Scheu, J. E. Kendrick, Y. Lavallée, and D. B. Dingwell, Universal scaling of fluid permeability during volcanic welding and sediment diagenesis, Geology 44, 219 (2016).

[5] P. Moldrup, T. Olesen, T. Komatsu, P. Schjønning, and D. E. Rolston, Tortuosity, diffusivity, and permeability in the soil liquid and gaseous phases, Soil Sci. Soc. Am. J. 65, 613 (2001).

[6] Z. Yang and A. Elgamal, Influence of permeability on liquefaction-induced shear deformation, J. Eng. Mech. 128, 720 (2002).

[7] J. Vasseur, F. B. Wadsworth, and D. B. Dingwell, Permeability of polydisperse magma foam, Geology 48, 536 (2020).

[8] J. Vasseur and F. B. Wadsworth, Sphere models for pore geometry and fluid permeability in heterogeneous magmas, Bull. Volcanol. 79, 77 (2017).

[9] M. D. M. Innocentini, V. R. Salvini, A. Macedo, and V. C. Pandolfelli, Prediction of ceramic foams permeability using Ergun's equation, Mater. Res. 2, 283 (1999).

[10] Darcy, Les Fontaines Publiques de La Ville de Dijon (Victor Dalmont, Paris, 1856).

[11] J. Kozeny, Über kapillare Leitung des Wassers im Boden, Sitzungsber. Akad. Wiss., Wien 136, 271 (1927).

[12] P. C. Carman, Fluid flow through granular beds, AIChE J. 15, 150 (1937).

[13] S. Ergun, Fluid flow through packed columns, Chem. Eng. Prog. 48, 89 (1952).

[14] I. F. Macdonald, M. S. El-Sayed, K. Mow, and F. A. L. Dullien, Flow through porous media-the Ergun equation revisited, Ind. Eng. Chem. Fundam. 18, 199 (1979).

[15] M. Röding, Z. Ma, and S. Torquato, Predicting permeability via statistical learning on higher-order microstructural information, Sci. Rep. 10, 15239 (2020).

[16] B. R. Corrochano, J. R. Melrose, A. C. Bentley, P. J. Fryer, and $\mathrm{S}$. Bakalis, A new methodology to estimate the steady-state permeability of roast and ground coffee in packed beds, J. Food Eng. 150, 106 (2015).
[17] H. Hasimoto, On the periodic fundamental solutions of the Stokes equations and their application to viscous flow past a cubic array of spheres, J. Fluid Mech. 5, 317 (1959).

[18] A. S. Sangani and A. Acrivos, Slow flow through a periodic array of spheres, Int. J. Multiphase Flow 8, 343 (1982).

[19] E. W. Llewellin, LBflow: An extensible lattice Boltzmann framework for the simulation of geophysical flows. Part II: Usage and validation, Comput. Geosci. 36, 123 (2010).

[20] M. D. Rintoul and S. Torquato, Reconstruction of the structure of dispersions, J. Colloid Interface Sci. 186, 467 (1997).

[21] E. Ghossein and M. Lévesque, A fully automated numerical tool for a comprehensive validation of homogenization models and its application to spherical particles reinforced composites, Int. J. Solids Struct. 49, 1387 (2012).

[22] E. Ghossein and M. Lévesque, Random generation of periodic hard ellipsoids based on molecular dynamics: A computationally-efficient algorithm, J. Comput. Phys. 253, 471 (2013).

[23] J. Segurado and J. Llorca, A numerical approximation to the elastic properties of sphere-reinforced composites, J. Mech. Phys. Solids 50, 2107 (2002).

[24] B. D. Lubachevsky and F. H. Stillinger, Geometric properties of random disk packings, J. Stat. Phys. 60, 561 (1990).

[25] B. D. Lubachevsky, F. H. Stillinger, and E. N. Pinson, Disks vs. spheres: Contrasting properties of random packings, J. Stat. Phys. 64, 501 (1991).

[26] S. Torquato, T. M. Truskett, and P. G. Debenedetti, Is Random Close Packing of Spheres Well Defined?, Phys. Rev. Lett. 84, 2064 (2000).

[27] K. W. Desmond and E. R. Weeks, Influence of particle size distribution on random close packing of spheres, Phys. Rev. E 90, 022204 (2014).

[28] T. Lewiner, H. Lopes, A. Vieira, and G. Tavares, Efficient implementation of marching cubes' cases with topological guarantees, J. Graph. Tools 8, 1 (2003).

[29] W. E. Lorensen and H. E. Cline, Marching cubes: A high resolution 3D surface construction algorithm, Comp. Graph. (ACM) 21, 163 (1987).

[30] E. W. Llewellin, LBflow: An extensible lattice Boltzmann framework for the simulation of geophysical flows. Part I: Theory and implementation, Comput. Geosci. 36, 115 (2010).

[31] F. B. Wadsworth, J. Vasseur, E. W. Llewellin, K. J. Dobson, M. Colombier, F. W. von Aulock, J. L. Fife, S. Wiesmaier, K.-U. Hess, B. Scheu, Y. Lavallée, and D. B. Dingwell, Topological inversions in coalescing granular media control fluid-flow regimes, Phys. Rev. E 96, 033113 (2017). 
[32] M. Matyka, Z. Koza, J. Gołembiewski, M. Kostur, and M. Januszewski, Anisotropy of flow in stochastically generated porous media, Phys. Rev. E 88, 023018 (2013).

[33] S. Torquato and B. Lu, Rigorous bounds on the fluid permeability: Effect of polydispersivity in grain size, Phys. Fluids A 2 , 487 (1990).

[34] N. S. Martys, S. Torquato, and D. P. Bentz, Universal scaling of fluid permeability for sphere packings, Phys. Rev. E 50, 403 (1994).

[35] F. B. Wadsworth, J. Vasseur, E. W. Llewellin, R. J. Brown, H. Tuffen, J. E. Gardner, J. E. Kendrick, Y. Lavallée, K. J. Dobson, M. J. Heap, D. B. Dingwell, K.-U. Hess, J. Schauroth, F. W. von Aulock, A. R. L. Kushnir, and F. Marone, A model for permeability evolution during volcanic welding, J. Volcanol. Geotherm. Res. 409, 107118 (2020).

[36] J. D. Blower, Factors controlling permeability-porosity relationships in magma, Bull. Volcanol. 63, 497 (2001).
[37] S. Feng, B. I. Halperin, and P. N. Sen, Transport properties of continuum systems near the percolation threshold, Phys. Rev. B 35, 197 (1987).

[38] B. I. Halperin, S. Feng, and P. N. Sen, Differences between Lattice and Continuum Percolation Transport Exponents, Phys. Rev. Lett. 54, 2391 (1985).

[39] M. D. Rintoul and S. Torquato, Precise determination of the critical threshold and exponents in a three-dimensional continuum percolation model, J. Phys. A: Math. Gen. 30, L585 (1997).

[40] F. B. Wadsworth, J. Vasseur, E. W. Llewellin, J. Schauroth, K. J. Dobson, B. Scheu, and D. B. Dingwell, Sintering of viscous droplets under surface tension, Proc. R. Soc. London, Ser. A 472, 20150780 (2016).

[41] S. Mueller, O. Melnik, O. Spieler, B. Scheu, and D. B. Dingwell, Permeability and degassing of dome lavas undergoing rapid decompression: An experimental determination, Bull. Volcanol. 67, 526 (2005). 